\title{
Trace operators on fractals, entropy and approximation numbers
}

\author{
Cornelia Schneider
}

\begin{abstract}
First we compute the trace space of Besov spaces $\mathfrak{B}_{p, q}^{s}$ - characterized via atomic decompositions - on fractals $\Gamma$, for parameters $0<p<\infty, 0<q \leq \min (1, p)$ and $s=(n-d) / p$. New Besov spaces $\mathcal{B}_{p, q}^{s}(\Gamma)$ on fractals are defined via traces for $0<p, q \leq \infty, s \geq(n-d) / p$ and some embedding assertions are established. We conclude by studying the compactness of the trace operator $\operatorname{Tr}_{\Gamma}$ by giving sharp estimates for entropy and approximation numbers of compact embeddings between Besov spaces. Our results on Besov spaces remain valid considering the classical spaces $\mathbf{B}_{p, q}^{s}$ defined via differences. The trace results are used to study traces in Triebel-Lizorkin spaces $\mathfrak{F}_{p, q}^{s}$ as well.
\end{abstract}

Keywords. Besov spaces, Triebel-Lizorkin spaces, $d$-sets, traces, entropy numbers, approximation numbers.

2010 Mathematics Subject Classification. 46E35, 47B06.

\section{Introduction}

The main object of this paper is to study the compactness of the trace operator $\operatorname{Tr}_{\Gamma}$ on fractals $\Gamma$ acting on Besov spaces $\mathfrak{B}_{p, q}^{s}$ (which we sometimes briefly denote as $\mathrm{B}$-spaces in the sequel). We use modern atomic decompositions to define the spaces $\mathfrak{B}_{p, q}^{s}\left(\mathbb{R}^{n}\right)$ as containing those $f \in L_{p}\left(\mathbb{R}^{n}\right)$ which can be represented as

$$
f(x)=\sum_{j=0}^{\infty} \sum_{m \in \mathbb{Z}^{n}} \lambda_{j, m} a_{j, m}(x), \quad x \in \mathbb{R}^{n},
$$

with coefficients $\lambda=\left\{\lambda_{j, m} \in \mathbb{C}: j \in \mathbb{N}_{0}, m \in \mathbb{Z}^{n}\right\}$ belonging to some appropriate sequence spaces $b_{p, q}^{s}$. In particular, $s>0,0<p \leq \infty, 0<q \leq \infty$, and $a_{j, m}(x)$ are normalized atoms. Furthermore,

$$
\left\|f\left|\mathfrak{B}_{p, q}^{s}\left(\mathbb{R}^{n}\right)\|:=\inf \| \lambda\right| b_{p, q}^{s}\right\|
$$

where the infimum is taken over all admissible representations $(0.1)$. 
We first extend the trace results obtained in [12] for hyperplanes $\mathbb{R}^{m}$ with $m<n$ (and generalized in [13] for domains) to the so-called $d$-sets $\Gamma$ introduced in Definition 2.1. Our assertions are collected in Theorem 2.7, where we obtain that for $0<p<\infty, 0<q \leq \min (1, p)$,

$$
\operatorname{Tr}_{\Gamma} \mathfrak{B}_{p, q}^{\frac{n-d}{p}}\left(\mathbb{R}^{n}\right)=L_{p}(\Gamma) .
$$

This together with the established embedding results for the spaces $\mathfrak{B}_{p, q}^{s}$ explains why it makes sense to speak about traces on $\Gamma$ for all spaces $\mathfrak{B}_{p, q}^{\sigma}\left(\mathbb{R}^{n}\right)$, where $\sigma>\frac{n-d}{p}$. All these previous considerations then allow us to introduce, in Definition 2.8, Besov spaces on fractals $\Gamma$ via traces,

$$
\mathcal{B}_{p, q}^{s}(\Gamma)=\operatorname{Tr}_{\Gamma} \mathfrak{B}_{p, q}^{s+\frac{n-d}{p}}\left(\mathbb{R}^{n}\right) .
$$

Embedding results for these new types of spaces are established.

Moreover, with the help of our trace results for Besov spaces, we are in a position to study traces on fractals for Triebel-Lizorkin spaces $\mathfrak{F}_{p, q}^{s}$ (sometimes briefly denoted as F-spaces) as well. The F-counterpart might even be more interesting here, since it turns out that in this case the traces are actually independent of the second parameter $q$.

The subsequent study of entropy and approximation numbers contributes to the question of the compactness of the trace operator $\operatorname{Tr}_{\Gamma}$ and related embeddings. We introduce entropy numbers $e_{k}$ and approximation numbers $a_{k}, k \in \mathbb{N}$, respectively, and establish some of their basic properties in the context of quasi-Banach spaces.

We will investigate entropy numbers of the trace operator $\operatorname{Tr}_{\Gamma}$ from $\mathfrak{B}_{p_{1}, q}^{s}\left(\mathbb{R}^{n}\right)$ into $L_{p_{2}}(\Gamma)$ - which is a compact map - and estimate

$$
e_{k}\left(\operatorname{Tr}_{\Gamma}: \mathfrak{B}_{p_{1}, q}^{s}\left(\mathbb{R}^{n}\right) \rightarrow L_{p_{2}}(\Gamma)\right) \sim k^{-\frac{s}{d}+\frac{n-d}{d p_{1}}},
$$

where $0<p_{1}, p_{2} \leq \infty, 0<q \leq \infty$, and $s-\frac{n-d}{p_{1}}>d \max \left(\frac{1}{p_{1}}-\frac{1}{p_{2}}, 0\right)$.

Although we mainly focus on entropy numbers, it is helpful to simultaneously have a close look at approximation numbers, for which we obtain

$$
a_{k}\left(\operatorname{Tr}_{\Gamma}: \mathfrak{B}_{p, p}^{s}\left(\mathbb{R}^{n}\right) \rightarrow L_{p}(\Gamma)\right) \sim k^{-\frac{s}{d}+\frac{n-d}{d p}} .
$$

Moreover, the connection between entropy and approximation numbers with eigenvalues yields further applications to spectral theory (which is outside the scope of this paper).

In particular, all our results for Besov spaces $\mathfrak{B}_{p, q}^{s}$ remain valid for the classical Besov spaces $\mathbf{B}_{p, q}^{s}$ defined via differences, as well. With some restrictions on the parameters this is also true for Triebel-Lizorkin spaces $\mathbf{F}_{p, q}^{s}$. 


\section{Preliminaries}

We use the standard notation. Let $\mathbb{N}$ be the collection of all natural numbers and let $\mathbb{N}_{0}=\mathbb{N} \cup\{0\}$. Let $\mathbb{R}^{n}$ be the Euclidean $n$-space, $n \in \mathbb{N}, \mathbb{C}$ the complex plane. The set of multi-indices $\beta=\left(\beta_{1}, \ldots, \beta_{n}\right), \beta_{i} \in \mathbb{N}_{0}, i=1, \ldots, n$, is denoted by $\mathbb{N}_{0}^{n}$, with $|\beta|=\beta_{1}+\cdots+\beta_{n}$, as usual. Moreover, if $x=\left(x_{1}, \ldots, x_{n}\right) \in \mathbb{R}^{n}$ and $\beta=\left(\beta_{1}, \ldots, \beta_{n}\right) \in \mathbb{N}_{0}^{n}$ we put $x^{\beta}=x_{1}^{\beta_{1}} \ldots x_{n}^{\beta_{n}}$.

We use the equivalence ' $\sim$ ' in

$$
a_{k} \sim b_{k} \quad \text { or } \quad \varphi(x) \sim \psi(x)
$$

always to mean that there are two positive numbers $c_{1}$ and $c_{2}$ such that

$$
c_{1} a_{k} \leq b_{k} \leq c_{2} a_{k} \quad \text { or } \quad c_{1} \varphi(x) \leq \psi(x) \leq c_{2} \varphi(x)
$$

for all admitted values of the discrete variable $k$ or the continuous variable $x$, where $\left\{a_{k}\right\}_{k},\left\{b_{k}\right\}_{k}$ are non-negative sequences and $\varphi, \psi$ are non-negative functions. If $a \in \mathbb{R}$, then $a_{+}:=\max (a, 0)$ and $[a]$ denotes the integer part of $a$.

Given two (quasi-) Banach spaces $X$ and $Y$, we write $X \hookrightarrow Y$ if $X \subset Y$ and the natural embedding of $X$ in $Y$ is continuous. All unimportant positive constants will be denoted by $c$, occasionally with subscripts. For convenience, let both $\mathrm{d} x$ and $|\cdot|$ stand for the ( $n$-dimensional) Lebesgue measure in the sequel. The space of all scalar-valued (real or complex), bounded and continuous functions on $\mathbb{R}^{n}$ is denoted by $C\left(\mathbb{R}^{n}\right)$, which is equipped with the $L_{\infty}\left(\mathbb{R}^{n}\right)$-norm.

Let $Q_{j, m}$ with $j \in \mathbb{N}_{0}$ and $m \in \mathbb{Z}^{n}$ denote a cube in $\mathbb{R}^{n}$ with sides parallel to the axes of coordinates, centered at $2^{-j} m$, and with side length $2^{-j+1}$. For a cube $Q$ in $\mathbb{R}^{n}$ and $r>0$, we denote by $r Q$ the cube in $\mathbb{R}^{n}$ concentric with $Q$ and with side length $r$ times the side length of $Q$. Furthermore, $\chi_{j, m}$ stands for the characteristic function of $Q_{j, m}$.

Function spaces on $\mathbb{R}^{\boldsymbol{n}}$. We give an atomic characterization of Besov and Triebel-Lizorkin spaces $\mathfrak{B}_{p, q}^{s}\left(\mathbb{R}^{n}\right)$ and $\mathfrak{F}_{p, q}^{s}\left(\mathbb{R}^{n}\right)$. This provides a constructive definition expanding functions $f$ via atoms (excluding any moment conditions) and suitable coefficients, where the latter belong to certain sequence spaces denoted by $b_{p, q}^{s}$ and $f_{p, q}^{s}$.

First we introduce the relevant sequence spaces.

Definition 1.1. Let $0<p \leq \infty, 0<q \leq \infty, s \in \mathbb{R}$, and

$$
\lambda=\left\{\lambda_{j, m} \in \mathbb{C}: j \in \mathbb{N}_{0}, m \in \mathbb{Z}^{n}\right\} .
$$


(i) Then

$$
b_{p, q}^{s}=\left\{\lambda:\left\|\lambda \mid b_{p, q}^{s}\right\|=\left(\sum_{j=0}^{\infty} 2^{j\left(s-\frac{n}{p}\right) q}\left(\sum_{m \in \mathbb{Z}^{n}}\left|\lambda_{j, m}\right|^{p}\right)^{q / p}\right)^{1 / q}<\infty\right\}
$$

(with the usual modification if $p=\infty$ and/or $q=\infty$ ).

(ii) Furthermore

$$
f_{p, q}^{s}=\left\{\lambda:\left\|\lambda\left|f_{p, q}^{s}\|=\|\left(\sum_{j=0}^{\infty} \sum_{m \in \mathbb{Z}^{n}} 2^{j s q}\left|\lambda_{j, m}\right|^{q} \chi_{j, m}(\cdot)\right)^{1 / q}\right| L_{p}\right\|<\infty\right\}
$$

Now we define the atoms.

Definition 1.2. Let $K \in \mathbb{N}_{0}$ and $r>1$. A $K$-times differentiable complex-valued function $a$ on $\mathbb{R}^{n}$ (continuous if $K=0$ ) is called a $K$-atom if for some $j \in \mathbb{N}_{0}$

$$
\text { supp } a \subset r Q_{j, m} \text { for some } m \in \mathbb{Z}^{n},
$$

and

$$
\left|\mathrm{D}^{\alpha} a(x)\right| \leq 2^{|\alpha| j} \quad \text { for }|\alpha| \leq K .
$$

It is convenient to write $a_{j, m}(x)$ instead of $a(x)$ if this atom is located at $Q_{j, m}$ according to (1.1). Furthermore, $K$ denotes the smoothness of the atom, cf. (1.2). We take the atomic characterization of function spaces of type $\mathfrak{B}_{p, q}^{s}\left(\mathbb{R}^{n}\right)$, $\mathfrak{F}_{p, q}^{s}\left(\mathbb{R}^{n}\right)$ as a definition.

Definition 1.3. Let $0<p \leq \infty, 0<q \leq \infty$, and $s>0$. Let $r>1$ and $K \in \mathbb{N}_{0}$ with

$$
K \geq(1+[s])
$$

be fixed.

(i) Then $f \in L_{p}\left(\mathbb{R}^{n}\right)$ belongs to $\mathfrak{B}_{p, q}^{s}\left(\mathbb{R}^{n}\right)$ if, and only if, it can be represented as

$$
f=\sum_{j=0}^{\infty} \sum_{m \in \mathbb{Z}^{n}} \lambda_{j, m} a_{j, m}(x),
$$

where $a_{j, m}$ are $K$-atoms $\left(j \in \mathbb{N}_{0}\right)$ with

$$
\operatorname{supp} a_{j, m} \subset r Q_{j, m}, \quad j \in \mathbb{N}_{0}, m \in \mathbb{Z}^{n},
$$

and $\lambda \in b_{p, q}^{s}$, convergence being in $L_{p}\left(\mathbb{R}^{n}\right)$. Furthermore,

$$
\left\|f\left|\mathfrak{B}_{p, q}^{s}\left(\mathbb{R}^{n}\right)\|=\inf \| \lambda\right| b_{p, q}^{s}\right\|,
$$

where the infimum is taken over all admissible representations (1.3). 
(ii) Then $f \in L_{p}\left(\mathbb{R}^{n}\right)$ belongs to $\mathfrak{F}_{p, q}^{s}\left(\mathbb{R}^{n}\right)$ if, and only if, it can be represented as

$$
f=\sum_{j=0}^{\infty} \sum_{m \in \mathbb{Z}^{n}} \lambda_{j, m} a_{j, m}(x),
$$

where the $a_{j, m}$ are $K$-atoms $\left(j \in \mathbb{N}_{0}\right)$ with

$$
\operatorname{supp} a_{j, m} \subset r Q_{j, m}, \quad j \in \mathbb{N}_{0}, m \in \mathbb{Z}^{n},
$$

and $\lambda \in f_{p, q}^{s}$, convergence being in $L_{p}\left(\mathbb{R}^{n}\right)$. Furthermore,

$$
\left\|f\left|\mathfrak{F}_{p, q}^{s}\left(\mathbb{R}^{n}\right)\|=\inf \| \lambda\right| f_{p, q}^{s}\right\|,
$$

where the infimum is taken over all admissible representations (1.5).

Remark 1.4. According to [18], based on [6], the spaces $\mathfrak{B}_{p, q}^{s}\left(\mathbb{R}^{n}\right)$ and $\mathfrak{F}_{p, q}^{s}\left(\mathbb{R}^{n}\right)$ are independent of $r$ and $K$. This may justify our omission of $K$ and $r$ in (1.4) and (1.6).

We will occasionally use an equivalent characterization of the Besov spaces $\mathfrak{B}_{p, q}^{s}\left(\mathbb{R}^{n}\right)$ using subatomic decompositions, which introduces $\mathfrak{B}_{p, q}^{s}\left(\mathbb{R}^{n}\right)$ as those $f \in L_{p}\left(\mathbb{R}^{n}\right)$ which can be represented as

$$
f(x)=\sum_{\beta \in \mathbb{N}_{0}^{n}} \sum_{j=0}^{\infty} \sum_{m \in \mathbb{Z}^{n}} \lambda_{j, m}^{\beta} k_{j, m}^{\beta}(x), \quad x \in \mathbb{R}^{n},
$$

with coefficients $\lambda=\left\{\lambda_{j, m}^{\beta} \in \mathbb{C}: \beta \in \mathbb{N}_{0}^{n}, j \in \mathbb{N}_{0}, m \in \mathbb{Z}^{n}\right\}$ belonging to some appropriate sequence space $b_{p, q}^{s, \varrho}$ defined as

$$
b_{p, q}^{s, \varrho}:=\left\{\lambda:\left\|\lambda \mid b_{p, q}^{s, \varrho}\right\|<\infty\right\}
$$

where

$$
\left\|\lambda \mid b_{p, q}^{s, \varrho}\right\|=\sup _{\beta \in \mathbb{N}_{0}^{n}} 2^{\varrho|\beta|}\left(\sum_{j=0}^{\infty} 2^{j(s-n / p) q}\left(\sum_{m \in \mathbb{Z}^{n}}\left|\lambda_{j, m}^{\beta}\right|^{p}\right)^{q / p}\right)^{1 / q},
$$

$s>0,0<p, q \leq \infty$ (with the usual modification if $p=\infty$ and/or $q=\infty$ ), $\varrho \geq 0$, and $k_{j, m}^{\beta}(x)$ are certain standardized building blocks. This subatomic characterization will turn out to be quite useful, in particular, when studying entropy numbers later on. The building blocks $k_{j, m}^{\beta}$ differ from the atoms $a$ in Definition 1.2 mainly by some unimportant technicalities. The advantage of building blocks is that they are universal. The price we pay for the universality is the extra 
summation over the additional parameter $\beta \in \mathbb{N}_{0}^{n}$ in (1.8) in contrast to Definition 1.3.

On the other hand when dealing with traces we prefer atoms over building blocks because their structure is much simpler and they provide a more qualitative description.

Moreover, the atomic approach for $B$-spaces is strongly linked with the classical approach which introduces $\mathbf{B}_{p, q}^{s}\left(\mathbb{R}^{n}\right)$ as subspaces of $L_{p}\left(\mathbb{R}^{n}\right)$ such that

$$
\left\|f\left|\mathbf{B}_{p, q}^{s}\left(\mathbb{R}^{n}\right)\left\|_{r}=\right\| f\right| L_{p}\left(\mathbb{R}^{n}\right)\right\|+\left(\int_{0}^{1} t^{-s q} \omega_{r}(f, t)_{p}^{q} \frac{\mathrm{d} t}{t}\right)^{1 / q}
$$

is finite, where $0<p \leq \infty, 0<q \leq \infty$ (with the usual modification if $q=\infty$ ), $s>0, r \in \mathbb{N}$ with $r>s$. Here $\omega_{r}(f, t)_{p}$ stands for the usual $r$-th modulus of smoothness of a function $f \in L_{p}\left(\mathbb{R}^{n}\right)$,

$$
\omega_{r}(f, t)_{p}=\sup _{|h| \leq t}\left\|\Delta_{h}^{r} f \mid L_{p}\left(\mathbb{R}^{n}\right)\right\|, \quad t>0,
$$

where

$$
\left(\Delta_{h}^{1} f\right)(x)=f(x+h)-f(x), \quad\left(\Delta_{h}^{r+1} f\right)(x)=\Delta_{h}^{1}\left(\Delta_{h}^{r} f\right)(x), \quad h \in \mathbb{R}^{n} .
$$

Recent results by Hedberg, Netrusov [6] on atomic decompositions and by Triebel [18, Section 9.2] on the reproducing formula prove the coincidences

$$
\mathbf{B}_{p, q}^{s}\left(\mathbb{R}^{n}\right)=\mathfrak{B}_{p, q}^{s}\left(\mathbb{R}^{n}\right), \quad s>0,0<p, q \leq \infty
$$

(in terms of equivalent quasi-norms). In particular, this implies that all our results for Besov spaces $\mathfrak{B}_{p, q}^{s}\left(\mathbb{R}^{n}\right)$ could as well be stated in terms of the classical spaces $\mathbf{B}_{p, q}^{s}\left(\mathbb{R}^{n}\right)$.

The following result will be needed later.

Proposition 1.5. Let $0<p, q \leq \infty, s>0, k \in \mathbb{N}$ with $k>s$, and let $h \in$ $C^{k}\left(\mathbb{R}^{n}\right)$. Then

$$
f \longrightarrow h f
$$

is a linear and bounded operator from $\mathfrak{B}_{p, q}^{s}\left(\mathbb{R}^{n}\right)$ into itself.

Proof. The atomic decomposition (1.3) multiplied by $h \in C^{k}$ gives an atomic decomposition of $h f$. In particular,

$$
\left\|h f\left|\mathfrak{B}_{p, q}^{s}\left(\mathbb{R}^{n}\right)\left\|\leq \sum_{|\alpha| \leq k} \sup _{x \in \mathbb{R}^{n}}\left|\mathrm{D}^{\alpha} h(x)\right| \cdot\right\| f\right| \mathfrak{B}_{p, q}^{s}\left(\mathbb{R}^{n}\right)\right\| .
$$


We summarize the embeddings for the spaces $\mathfrak{B}_{p, q}^{s}\left(\mathbb{R}^{n}\right)$, cf. [5].

Proposition 1.6. Let $0<s_{2}<s_{1}, 0<p_{1} \leq p_{2} \leq \infty$, and $0<q_{1}, q_{2} \leq \infty$. If

$$
\delta=s_{1}-s_{2}-n\left(\frac{1}{p_{1}}-\frac{1}{p_{2}}\right)>0,
$$

we have the embedding

$$
\mathfrak{B}_{p_{1}, q_{1}}^{s_{1}}\left(\mathbb{R}^{n}\right) \hookrightarrow \mathfrak{B}_{p_{2}, q_{2}}^{s_{2}}\left(\mathbb{R}^{n}\right) .
$$

Remark 1.7. In terms of boundedness it was shown in [5] that

$$
\mathfrak{B}_{p, q}^{n / p}\left(\mathbb{R}^{n}\right) \hookrightarrow L_{\infty}\left(\mathbb{R}^{n}\right) \quad \text { if and only if } \quad 0<p<\infty, 0<q \leq 1 \text {. }
$$

Together with Proposition 1.6 we see that

$$
\mathfrak{B}_{p, q}^{s}\left(\mathbb{R}^{n}\right) \hookrightarrow L_{\infty}\left(\mathbb{R}^{n}\right), \quad s>\frac{n}{p}, 0<p, q \leq \infty .
$$

In particular, $L_{\infty}$ can be replaced by $C$ in (1.11) and (1.12).

\section{Traces on fractals $\Gamma$}

In this section we want to establish trace results for B- and F-spaces on $d$-sets $\Gamma$. We use the convention to let $\mathfrak{U}_{p, q}^{s}$ denote one of the spaces $\mathfrak{B}_{p, q}^{s}$ and $\mathfrak{F}_{p, q}^{s}$, respectively. We briefly explain our understanding of the trace operator. Recall that for $x=\left(x^{\prime}, x_{n}\right) \in \mathbb{R}^{n}$ with $x^{\prime} \in \mathbb{R}^{n-1}$ the mapping

$$
\operatorname{Tr}_{\mathbb{R}^{n-1}}: \varphi(x) \longrightarrow \varphi\left(x^{\prime}, 0\right), \quad \varphi \in S\left(\mathbb{R}^{n}\right),
$$

is called the trace of $\varphi$ on $\mathbb{R}^{n-1}$. We explain the fractal counterpart of (2.1) now. Let us temporarily consider a closed set $\Gamma \subset \mathbb{R}^{n}$ with $|\Gamma|=0$ and assume that there exists a Radon measure $\mu$ on $\mathbb{R}^{n}$ with supp $\mu=\Gamma$, and let $L_{p}(\Gamma)$ be the related $L_{p}$-spaces on $\Gamma$. Then the restriction $\operatorname{Tr}_{\Gamma}=\left.\varphi\right|_{\Gamma}$ understood pointwise is well-defined for any $\varphi \in S\left(\mathbb{R}^{n}\right)$. In fact, the restriction of a smooth function to a subset $\Gamma \subset \mathbb{R}^{n}$ is always meaningful. The Radon measure on $\Gamma$ ensures that $\left.\varphi\right|_{\Gamma}$ makes sense in $L_{p}(\Gamma)$. Moreover, suppose for $s>0$ and $0<p, q<\infty$ there is a constant $c>0$ such that

$$
\left\|\operatorname{Tr}_{\Gamma} \varphi\left|L_{p}(\Gamma)\|\leq c\| \varphi\right| \mathfrak{U}_{p, q}^{s}\left(\mathbb{R}^{n}\right)\right\| \quad \text { for all } \varphi \in S\left(\mathbb{R}^{n}\right) .
$$

Since the Schwartz space $S\left(\mathbb{R}^{n}\right)$ is dense in $\mathfrak{U}_{p, q}^{s}\left(\mathbb{R}^{n}\right)$, the inequality (2.2) may be extended by completion to all $f \in \mathfrak{U}_{p, q}^{s}\left(\mathbb{R}^{n}\right)$. The resulting limit will be denoted 
by $\operatorname{Tr}_{\Gamma} f$. Note that it is independent of the approximation of $f \in \mathfrak{A}_{p, q}^{s}\left(\mathbb{R}^{n}\right)$ by functions $\varphi_{j} \in S\left(\mathbb{R}^{n}\right)$ due to (2.2). In addition, the equality

$$
\operatorname{Tr}_{\Gamma} \mathfrak{U}_{p, q}^{s}\left(\mathbb{R}^{n}\right)=L_{p}(\Gamma)
$$

must be understood in the sense that any $f^{\Gamma} \in L_{p}(\Gamma)$ is the trace in the above sense of a suitable $g \in \mathfrak{U}_{p, q}^{s}\left(\mathbb{R}^{n}\right)$ on $\Gamma$ and $\left\|f^{\Gamma} \mid L_{p}(\Gamma)\right\|$ is equivalent to

$$
\inf \left\{\left\|g \mid \mathfrak{A}_{p, q}^{s}\left(\mathbb{R}^{n}\right)\right\|: \operatorname{Tr}_{\Gamma} g=f^{\Gamma}\right\} .
$$

We mention explicitly that (2.3) does not mean that there is a linear extension operator from $L_{p}(\Gamma)$ into $\mathfrak{U}_{p, q}^{s}\left(\mathbb{R}^{n}\right)$. This is not so, even not for $\Gamma=\mathbb{R}^{n-1}$, cf. [12, Remark 3.3]. But it will not be necessary when studying entropy numbers in the next section since they are nonlinear by nature.

We now define the $d$-sets on which we wish to study trace operators in the sequel.

Definition 2.1. A set $\Gamma \subset \mathbb{R}^{n}$ is called $d$-set, $0<d<n$, if there exists a compact Radon measure $\mu$ in $\mathbb{R}^{n}$ such that $\operatorname{supp} \mu=\Gamma$ and there are constants $c_{1}, c_{2}>0$ such that for arbitrary $\gamma \in \Gamma$ and all $0<r<1$ we have

$$
c_{1} r^{d} \leq \mu(B(\gamma, r) \cap \Gamma) \leq c_{2} r^{d} .
$$

Remark 2.2. Note that some self-similar fractals are outstanding examples of $d$-sets. For instance, the usual (middle-third) Cantor set in $\mathbb{R}$ is a $d$-set for $d=\ln 2 / \ln 3$, and the Koch curve in $\mathbb{R}^{2}$ is a $d$-set for $d=\ln 4 / \ln 3$. It is well known that $\left.\mu \sim \mathscr{H}^{d}\right|_{\Gamma}$, the restriction of the $d$-dimensional Hausdorff measure to $\Gamma$, cf. [15, Chapter 1].

An important property of $d$-sets that is useful for studying trace operators is given below.

Definition 2.3. A compact set $\Gamma \subset \mathbb{R}^{n}$ is said to be porous if there is a number $\eta$ with $0<\eta<1$, such that one finds, for any ball $B(x, r)$ with radius $0<r<1$, a ball $B(y, \eta r)$ with

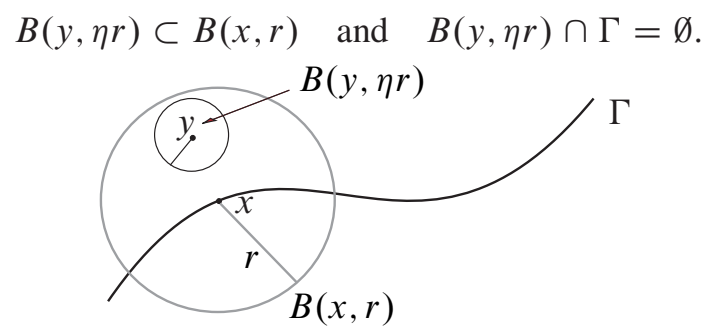


Remark 2.4. For details we refer to [16, Sections 9.16-9.19], where this property was called the ball condition. In particular, any $d$-set $\Gamma$ with $d<n$ is porous. A proof may be found in [16, Section 9.18]. Furthermore, if a set $\Gamma$ is porous, then $|\Gamma|=0$.

By analogy with Definition 1.1 we now define the corresponding sequence spaces on compact sets $\Gamma$ making use of the abbreviation

$$
\sum_{m \in \mathbb{Z}^{n}}^{\Gamma, j}=\sum_{m \in \mathbb{Z}^{n}, r Q_{j, m} \cap \Gamma \neq \emptyset} .
$$

Definition 2.5. Let $0<p \leq \infty, 0<q \leq \infty, s \in \mathbb{R}$, and

$$
\lambda=\left\{\lambda_{j, m} \in \mathbb{C}: j \in \mathbb{N}_{0}, m \in \mathbb{Z}^{n}\right\} .
$$

(i) Then

$$
\begin{aligned}
b_{p, q}^{s}(\Gamma)=\{\lambda & :\left\|\lambda \mid b_{p, q}^{s}(\Gamma)\right\| \\
& \left.=\left(\sum_{j=0}^{\infty} 2^{j\left(s-\frac{n}{p}\right) q}\left(\sum_{m \in \mathbb{Z}^{n}}^{\Gamma, j}\left|\lambda_{j, m}\right|^{p}\right)^{q / p}\right)^{1 / q}<\infty\right\}
\end{aligned}
$$

(with the usual modification if $p=\infty$ and/or $q=\infty$ ).

(ii) Furthermore

$$
\begin{aligned}
f_{p, q}^{s}(\Gamma)=\{\lambda & :\left\|\lambda \mid f_{p, q}^{s}(\Gamma)\right\| \\
& \left.=\left\|\left(\sum_{j=0}^{\infty} \sum_{m \in \mathbb{Z}^{n}}^{\Gamma, j} 2^{j s q}\left|\lambda_{j, m}\right|^{q} \chi_{j, m}(\cdot)\right)^{1 / q} \mid L_{p}\left(\mathbb{R}^{n}\right)\right\|<\infty\right\} .
\end{aligned}
$$

The following proposition will be quite useful when studying traces in TriebelLizorkin spaces. A proof may be found in [18, Proposition 9.22].

Proposition 2.6. Let $0<p<\infty, 0<q \leq \infty, s \in \mathbb{R}$, and let $\Gamma$ be a compact porous set in $\mathbb{R}^{n}$. Then $f_{p, q}^{s}(\Gamma)$ is independent of $q$. In particular,

$$
f_{p, q}^{s}(\Gamma)=b_{p, p}^{s}(\Gamma) .
$$

Trace results. We now present the main theorem concerning traces on $d$-sets $\Gamma$ for Besov and Triebel-Lizorkin spaces of type $\mathfrak{U}_{p, q}^{s}\left(\mathbb{R}^{n}\right)$. In particular, we first establish trace results for the spaces $\mathfrak{B}_{p, q}^{s}\left(\mathbb{R}^{n}\right)$, which then will help us to 
investigate the trace problem in $\mathfrak{F}_{p, q}^{s}\left(\mathbb{R}^{n}\right)$. It turns out that in the latter case the trace is actually independent of the parameter $q$.

Theorem 2.7. Let $\Gamma$ be a $d$-set with $0<d<n$. Let $0<p<\infty$ and $0<q \leq \infty$.

(i) Then

$$
\operatorname{Tr}_{\Gamma} \mathfrak{B}_{p, q}^{\frac{n-d}{p}}\left(\mathbb{R}^{n}\right)=L_{p}(\Gamma), \quad 0<q \leq \min (1, p) .
$$

(ii) Furthermore,

$$
\operatorname{Tr}_{\Gamma} \mathfrak{F}_{p, q}^{\frac{n-d}{p}}\left(\mathbb{R}^{n}\right)=L_{p}(\Gamma), \quad 0<p \leq 1 .
$$

Proof. We fix some notation. Let $\left.D\right|_{\Gamma}$ be the restriction of $D\left(\mathbb{R}^{n}\right)$ to $\Gamma$. Our proof is based upon the ideas found in [15, Theorem 18.6]. We essentially rely on atomic decomposition techniques (where we have no restrictions in terms of moment conditions here).

Step 1. Let $0<p<\infty$ and $0<q \leq \min (1, p)$. In this step it is proved that

$$
\operatorname{Tr}_{\Gamma} \mathfrak{B}_{p, q}^{\frac{n-d}{p}}\left(\mathbb{R}^{n}\right) \subset L_{p}(\Gamma) .
$$

We use an optimal atomic decomposition of $\varphi \in S\left(\mathbb{R}^{n}\right)$ in $\mathfrak{B}_{p, q}^{\frac{n-d}{p}}\left(\mathbb{R}^{n}\right)$ according to Definition 1.3, i.e.,

$$
\varphi(x)=\sum_{j, m} \lambda_{j, m} a_{j, m}(x) \quad \text { and } \quad\left\|\lambda\left|b_{p, q}^{\frac{n-d}{p}}\|\leq c\| \varphi\right| \mathfrak{B}_{p, q}^{\frac{n-d}{p}}\left(\mathbb{R}^{n}\right)\right\|,
$$

where $c>0$ is independent of $\varphi$ and $a_{j, m}$ are suitable atoms according to Definition 1.2 .

Let $0<p \leq 1$. By the support property (1.1) of $a_{j, m}$ and (2.4) it follows that

$$
\begin{aligned}
\left\|\operatorname{Tr}_{\Gamma} \varphi \mid L_{p}(\Gamma)\right\|^{p} & \leq \sum_{j=0}^{\infty} \int_{\Gamma}\left|\sum_{m \in \mathbb{Z}^{n}} \lambda_{j, m} a_{j, m}(\gamma)\right|^{p} \mu(\mathrm{d} \gamma) \\
& \leq c \sum_{j, m}\left|\lambda_{j, m}\right|^{p} 2^{-d j}=c \sum_{j} 2^{j\left(\frac{n-d}{p}-\frac{n}{p}\right) p} \sum_{m}\left|\lambda_{j, m}\right|^{p} \\
& \leq c\left(\sum_{j} 2^{j\left(\frac{n-d}{p}-\frac{n}{p}\right) q}\left(\sum_{m}\left|\lambda_{j, m}\right|^{p}\right)^{q / p}\right)^{p / q} \\
& =c\left\|\lambda \mid b_{p, q}^{\frac{n-d}{p}}\right\|^{p},
\end{aligned}
$$

where we have used $q \leq p$. Together with (2.8) we obtain (2.7) by completion. If $p>1$, then the first inequality in (2.9) must be replaced by the usual triangle inequality, and one has to use $q \leq 1$. 
Step 2. Let $0<p<\infty$ and $0<q \leq \min (1, p)$. We give a proof of the reverse inclusion

$$
L_{p}(\Gamma) \subset \operatorname{Tr}_{\Gamma} \mathfrak{B}_{p, q}^{\frac{n-d}{p}}\left(\mathbb{R}^{n}\right) .
$$

It is known that $\left.D\right|_{\Gamma}$ is dense in $L_{p}(\Gamma)$, cf. [15, Theorem 3.8] and without loss of generality we may work with $\varphi \in D\left(\mathbb{R}^{n}\right)$. Moreover, we assume that $\left.\varphi\right|_{\Gamma} \neq 0$. Consider the neighborhood of $\Gamma$ given by

$$
\Gamma_{k}=\left\{x \in \mathbb{R}^{n}: \operatorname{dist}(x, \Gamma)<2^{-k}\right\} .
$$

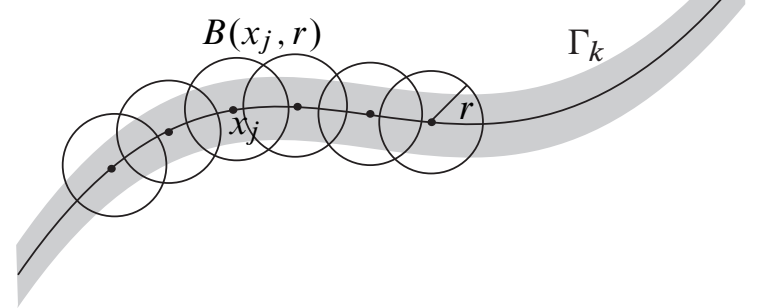

Since $\Gamma$ is compact, together with the properties of the Hausdorff measure, there are open balls $B\left(x_{j}, r\right)$ with $j=1, \ldots, N$ centered at $\Gamma$ with the same radius $r>0$ that cover $\Gamma$. Note that

$$
\bar{\Gamma}_{k} \subset \bigcup_{j=1}^{N} B\left(x_{j}, r\right),
$$

where $k$ depends on the given covering.

Now let $\left\{\varphi_{j}\right\}_{j=1}^{N}$ be a smooth resolution of unity in a neighborhood $\Gamma_{k}$ of $\Gamma \cap$ $\operatorname{supp} \varphi$ adapted to $\left(B\left(x_{j}, r\right)\right)_{j=1}^{N}$. In particular, we have

$$
\sum_{j=1}^{N} \varphi_{j}(x)=1 \quad \text { for } x \in \Gamma_{k} \text { and } \operatorname{supp} \varphi_{j} \subset B\left(x_{j}, r\right) .
$$

Let $\lambda_{j}:=\max _{x \in B\left(x_{j}, r\right)}|\varphi(x)|$. Then

$$
\varphi(x)=\sum_{j=1}^{N} \varphi(x) \varphi_{j}(x)=\sum_{j=1}^{N} \lambda_{j}\left[\lambda_{j}^{-1} \varphi(x) \varphi_{j}(x)\right], \quad x \in \Gamma_{k},
$$

where the terms with $\lambda_{j}=0$ are omitted. Put

$$
a_{j}(x)=\lambda_{j}^{-1} \varphi(x) \varphi_{j}(x) .
$$


We have supp $a_{j} \subset B\left(x_{j}, r\right)$. Furthermore, in dependence on $\varphi$ we choose $r \sim 2^{-l}>0$ small enough (hence $\left\{\varphi_{j}\right\}$ is chosen in dependence on $\varphi$ as well). We see that

$$
\left|a_{j}(x)\right|=\frac{|\varphi(x)|}{\lambda_{j}}\left|\varphi_{j}(x)\right| \leq c^{\prime}
$$

and analogous estimates hold for all $\mathrm{D}^{\alpha} a_{j}$. Hence (2.11) provides a suitable atomic decomposition. For all $\varphi \in D\left(\mathbb{R}^{n}\right)$ we therefore have

$$
\begin{aligned}
\left\|\varphi \mid \mathfrak{B}_{p, q}^{\frac{n-d}{p}}\left(\mathbb{R}^{n}\right)\right\| & \leq\left\|\lambda \mid b_{p, q}^{\frac{n-d}{p}}\right\| \sim 2^{l\left(\frac{n-d}{p}-\frac{n}{p}\right)}\left(\sum_{j=1}^{N}\left|\lambda_{j}\right|^{p}\right)^{1 / p} \\
& =\left(2^{-l d} \sum_{j=1}^{N}\left|\lambda_{j}\right|^{p}\right)^{1 / p} \sim\left(r^{d} \sum_{j=1}^{N}\left|\lambda_{j}\right|^{p}\right)^{1 / p} \\
& \leq c^{\prime}\left\|\operatorname{Tr}_{\Gamma} \varphi \mid L_{p}(\Gamma)\right\|,
\end{aligned}
$$

where the last step follows from the assumption that $\Gamma$ is a $d$-set, i.e., by (2.4) we have $r^{d} \sim \mu(B(\gamma, r) \cap \Gamma)$, and the fact that $r>0$ can be chosen arbitrarily small.

It follows from the density of $\left.D\right|_{\Gamma}$ in $L_{p}(\Gamma)$ that any nontrivial $f \in L_{p}(\Gamma)$ can be represented in the form

$$
f(\gamma)=\sum_{j=1}^{\infty} f_{j}(\gamma), \quad \gamma \in \Gamma, f_{j} \in D\left(\mathbb{R}^{n}\right),
$$

with

$$
0<\left\|\operatorname{Tr}_{\Gamma} f_{j}\left|L_{p}(\Gamma)\left\|\leq c 2^{-j}\right\| f\right| L_{p}(\Gamma)\right\|, \quad j \in \mathbb{N} .
$$

By (2.12) we have

$$
\left\|f_{j}\left|\mathfrak{B}_{p, q}^{\frac{n-d}{p}}\left(\mathbb{R}^{n}\right)\left\|\leq c^{\prime}\right\| \operatorname{Tr}_{\Gamma} f_{j}\right| L_{p}(\Gamma)\right\| .
$$

Now we may define an extension operator

$$
\operatorname{Ex} f=\sum_{j=1}^{\infty} f_{j} \in \mathfrak{B}_{p, q}^{\frac{n-d}{p}}\left(\mathbb{R}^{n}\right), \quad \operatorname{Tr}_{\Gamma} \operatorname{Ex} f=f,
$$

and obtain by virtue of (2.13) and (2.14),

$$
\left\|\operatorname{Ex} f\left|\mathfrak{B}_{p, q}^{\frac{n-d}{p}}\left(\mathbb{R}^{n}\right)\left\|\leq c^{\prime}\right\| f\right| L_{p}(\Gamma)\right\|,
$$

which proves (2.10). 
Step 3. We prove (ii). Assume that $\operatorname{Tr}_{\Gamma} \mathfrak{F}_{p, q}^{\frac{n-d}{p}}\left(\mathbb{R}^{n}\right)$ is independent of $q$. Then (2.6) including the equivalence of the two involved quasi-norms follows from (2.5) with $p=q \leq 1$ since $\mathfrak{B}_{p, p}^{s}\left(\mathbb{R}^{n}\right)=\mathfrak{F}_{p, p}^{s}\left(\mathbb{R}^{n}\right)$. In order to prove the independence of the trace on $\Gamma$ of $q$ we first observe that

$$
\operatorname{Tr}_{\Gamma} \mathfrak{F}_{p, q_{1}}^{\frac{n-d}{p}}\left(\mathbb{R}^{n}\right) \subset \operatorname{Tr}_{\Gamma} \mathfrak{F}_{p, q_{2}}^{\frac{n-d}{p}}\left(\mathbb{R}^{n}\right) \quad \text { if } 0<q_{1} \leq q_{2} \leq \infty
$$

follows simply by the monotonicity of the two spaces. To show the reverse inclusion

$$
\operatorname{Tr}_{\Gamma} \mathfrak{F}_{p, q_{2}}^{\frac{n-d}{p}}\left(\mathbb{R}^{n}\right) \subset \operatorname{Tr}_{\Gamma} \mathfrak{F}_{p, q_{1}}^{\frac{n-d}{p}}\left(\mathbb{R}^{n}\right)
$$

we choose an optimal atomic decomposition for $f \in \mathfrak{F}_{p, q_{2}}^{\frac{n-d}{p}}\left(\mathbb{R}^{n}\right)$, i.e.,

$$
f(x)=\sum_{j=0}^{\infty} \sum_{m \in \mathbb{Z}^{n}} \lambda_{j, m} a_{j, m}(x) \quad \text { and } \quad\left\|\lambda\left|f_{p, q_{2}}^{\frac{n-d}{p}}\|\sim\| f\right| \mathfrak{F}_{p, q_{2}}^{\frac{n-d}{p}}\left(\mathbb{R}^{n}\right)\right\| .
$$

Let

$$
f_{\Gamma}(x)=\sum_{j} \sum_{m \in \mathbb{Z}^{n}}^{\Gamma, j} \lambda_{j, m} a_{j, m}(x),
$$

where the summation in (2.16) is restricted to those $m$ (in dependence on $j$ ) such that

$$
\operatorname{supp} a_{j, m} \cap \Gamma \neq \varnothing \text {. }
$$

Of course,

$$
\operatorname{Tr}_{\Gamma} f=\operatorname{Tr}_{\Gamma} f_{\Gamma} .
$$

Using Proposition 2.6 it follows that $f_{\Gamma} \in \mathfrak{F}_{p, q_{1}}^{\frac{n-d}{p}}\left(\mathbb{R}^{n}\right)$ since

$$
\begin{aligned}
\left\|f_{\Gamma} \mid \mathfrak{F}_{p, q_{1}}^{\frac{n-d}{p}}\left(\mathbb{R}^{n}\right)\right\| & \leq\left\|\lambda\left|f_{p, q_{1}}^{\frac{n-d}{p}}(\Gamma)\|=\| \lambda\right| f_{p, q_{2}}^{\frac{n-d}{p}}(\Gamma)\right\| \\
& \leq\left\|\lambda\left|f_{p, q_{2}}^{\frac{n-d}{p}}\|\sim\| f\right| \mathfrak{F}_{p, q_{2}}^{\frac{n-d}{p}}\left(\mathbb{R}^{n}\right)\right\|<\infty .
\end{aligned}
$$

This finally completes the proof.

Function spaces on fractals $\boldsymbol{\Gamma}$. Instead of trying to introduce intrinsically spaces $\mathfrak{B}_{p, q}^{s}(\Gamma)$ as is often done for function spaces on domains $\Omega \subset \mathbb{R}^{n}$, we define the corresponding spaces on fractals $\Gamma$ via traces and study their properties. Let $\Gamma$ be a $d$-set in $\mathbb{R}^{n}$ according to Definition 2.1 with $0<d<n$. By (2.7) for any $0<p<\infty$ and $\bar{p}=\min (1, p)$ we have

$$
\operatorname{Tr}_{\Gamma} \mathfrak{B}_{p, \bar{p}}^{\frac{n-d}{p}}\left(\mathbb{R}^{n}\right) \subset L_{p}(\Gamma)
$$


and

$$
\left\|\operatorname{Tr}_{\Gamma} f\left|L_{p}(\Gamma)\|\leq c\| f\right| \mathfrak{B}_{p, \bar{p}}^{\frac{n-d}{p}}\left(\mathbb{R}^{n}\right)\right\|
$$

for some $c>0$ and all $f \in \mathfrak{B}_{p, \bar{p}}^{\frac{n-d}{p}}\left(\mathbb{R}^{n}\right)$. Therefore it makes sense to speak about traces on $\Gamma$ for all spaces $\mathfrak{B}_{p, q}^{\sigma}\left(\mathbb{R}^{n}\right)$ with $0<p<\infty, 0<q \leq \infty$ and $\sigma>\frac{n-d}{p}$ as subspaces of $L_{p}(\Gamma)$. We may extend our considerations as well to $p=\infty$. In that case, $\mathfrak{B}_{\infty, q}^{\sigma}\left(\mathbb{R}^{n}\right)$ consists of continuous functions and the trace is taken pointwise, cf. Remark 1.7.

With these previous considerations it makes sense to introduce Besov spaces on fractals with the help of traces as follows.

Definition 2.8. Let $\Gamma$ be a $d$-set in $\mathbb{R}^{n}$ according to Definition 2.1 with $0<d<n$. Let $s>0$ and $0<p, q \leq \infty$. Then

$$
\mathscr{B}_{p, q}^{s}(\Gamma)=\operatorname{Tr}_{\Gamma} \mathfrak{B}_{p, q}^{s+\frac{n-d}{p}}\left(\mathbb{R}^{n}\right),
$$

equipped with the quasi-norm

$$
\left\|f\left|\mathscr{B}_{p, q}^{s}(\Gamma)\|=\inf \| g\right| \mathfrak{B}_{p, q}^{s+\frac{n-d}{p}}\left(\mathbb{R}^{n}\right)\right\|,
$$

where the infimum is taken over all $g \in \mathfrak{B}_{p, q}^{s+\frac{n-d}{p}}\left(\mathbb{R}^{n}\right)$ with $\operatorname{Tr}_{\Gamma} g=f$.

Remark 2.9. (i) By standard arguments $\mathscr{B}_{p, q}^{s}(\Gamma)$ is a quasi-Banach space (a Banach space if $p \geq 1, q \geq 1)$ always considered as a subspace of $L_{p}(\Gamma)$ according to (2.17) and (2.18).

(ii) Let $m \in \mathbb{N}$ with $m>n$ and let $\mathbb{R}^{n}$ be interpreted as a hyperplane in $\mathbb{R}^{m}$. For $s>0$ we have

$$
\operatorname{Tr}_{\mathbb{R}^{n}} \mathfrak{B}_{p, q}^{s+\frac{m-d}{p}}\left(\mathbb{R}^{m}\right)=\mathfrak{B}_{p, q}^{s+\frac{n-d}{p}}\left(\mathbb{R}^{n}\right),
$$

cf. [12, Remark 3.4]. Now it follows easily that $\mathcal{B}_{p, q}^{s}(\Gamma)$ is independent of the dimension of the underlying space $\mathbb{R}^{n}$.

(iii) Definition 2.8 is a natural generalization of the well-known trace results on hyperplanes, cf. [12]. Let $\Gamma=\mathbb{R}^{d}, d<n$, interpreted as a hyperplane in $\mathbb{R}^{n}$. Then [12, Remark 3.4] yields

$$
\operatorname{Tr}_{\mathbb{R}^{d}} \mathfrak{B}_{p, q}^{s+\frac{n-d}{p}}\left(\mathbb{R}^{n}\right)=\mathfrak{B}_{p, q}^{s}\left(\mathbb{R}^{d}\right)
$$

and in this context

$$
\mathfrak{B}_{p, q}^{s}\left(\mathbb{R}^{d}\right)=\mathfrak{B}_{p, q}^{s}\left(\mathbb{R}^{d}\right)
$$


(iv) Compare the spaces $\mathscr{B}_{p, q}^{s}(\Gamma)$ from Definition 2.8 with the spaces

$$
\mathbb{B}_{p, q}^{s}(\Gamma)=\operatorname{Tr}_{\Gamma} B_{p, q}^{s+\frac{n-d}{p}}\left(\mathbb{R}^{n}\right)
$$

introduced in [15, p. 159]. Obviously, these spaces coincide when

$$
s+\frac{n-d}{p}>n\left(\frac{1}{p}-1\right)_{+} .
$$

Furthermore, it might actually be that they coincide for all $s>0$ since when dealing with hyperplanes $\mathbb{R}^{d}$, we have

$$
\operatorname{Tr}_{\mathbb{R}^{d}} B_{p, q}^{s+\frac{n-d}{p}}\left(\mathbb{R}^{n}\right)=\mathfrak{B}_{p, q}^{s}\left(\mathbb{R}^{d}\right)
$$

and

$$
\operatorname{Tr}_{\mathbb{R}^{d}} \mathfrak{B}_{p, q}^{s+\frac{n-d}{p}}\left(\mathbb{R}^{n}\right)=\mathfrak{B}_{p, q}^{s}\left(\mathbb{R}^{d}\right),
$$

cf. [12, Theorem 3.10]. One would need to check in the general case whether spaces $\mathfrak{B}_{p, q}^{s}(\Gamma)$ defined intrinsically on $d$-sets $\Gamma$ coincide with the trace spaces

$$
\operatorname{Tr}_{\Gamma} \mathfrak{B}_{p, q}^{s+\frac{n-d}{p}}\left(\mathbb{R}^{n}\right) .
$$

But this is out of our focus now. Nevertheless, it makes sense to introduce the spaces $\mathscr{B}_{p, q}^{s}(\Gamma)$ by themselves - in contrast to the spaces $\mathbb{B}_{p, q}^{s}(\Gamma)$ - since they both arise from a different context. In particular $B_{p, q}^{s}\left(\mathbb{R}^{n}\right)$ and $\mathfrak{B}_{p, q}^{s}\left(\mathbb{R}^{n}\right)$ cannot be compared if $0<s \leq n\left(\frac{1}{p}-1\right), 0<p<1$. Details may be found in [14, Section 2] and [11].

(v) Furthermore, it should be possible to extend our results to $(d, \psi)$-sets as was done in a similar context in [8]. But this study is postponed till a later occasion.

In terms of embeddings for $\mathscr{B}_{p, q}^{s}(\Gamma)$ we have the following results.

Proposition 2.10. Let $0<s_{2}<s_{1}<\infty, 0<p_{1}, p_{2} \leq \infty$, and $0<q_{1}, q_{2} \leq$ $\infty$. If

$$
\delta_{+}=s_{1}-s_{2}-d\left(\frac{1}{p_{1}}-\frac{1}{p_{2}}\right)_{+}>0,
$$

we have the embedding

$$
\mathcal{B}_{p_{1}, q_{1}}^{s_{1}}(\Gamma) \hookrightarrow \mathcal{B}_{p_{2}, q_{2}}^{s_{2}}(\Gamma)
$$


Proof. If $p_{1}>p_{2}$, the embedding

$$
\mathcal{B}_{p_{1}, q_{2}}^{s_{2}}(\Gamma) \hookrightarrow \mathscr{B}_{p_{2}, q_{2}}^{s_{2}}(\Gamma)
$$

may be proved using building blocks as was done in [8, Proposition 2.18] for similar spaces. Also, when $p_{1} \leq p_{2}$, by the same arguments the embedding results from Proposition 1.6 carry over.

In the diagram below we have sketched the maximal area of possible embeddings of a fixed original space $\mathcal{B}_{p_{1}, q_{1}}^{s_{1}}(\Gamma)$ into spaces $\mathcal{B}_{p_{2}, q_{2}}^{s_{2}}(\Gamma), \mathcal{B}_{p_{3}, q_{3}}^{s_{3}}(\Gamma)$. The lighter shaded area corresponds to the additional embeddings we have since the fractal $\Gamma$ is bounded.

The corresponding assertions for Besov spaces of type $B_{p, q}^{s}(\Omega)$ on bounded domains $\Omega \subset \mathbb{R}^{n}$ may be found in [14, 3.3.1].

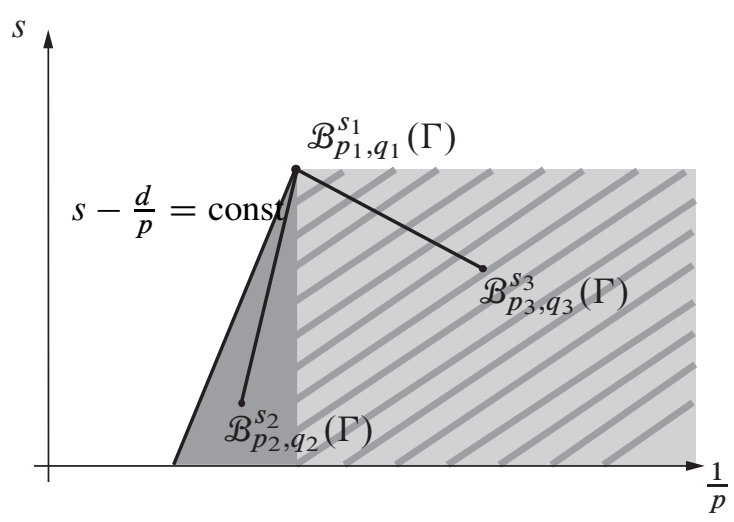

\section{Entropy and approximation numbers}

The main aim of this section is to study the compactness of the trace operator. More precisely, we use the known results on entropy numbers to investigate the behavior of those numbers of compact embeddings between Besov spaces $\mathfrak{B}_{p, q}^{s}$. In particular, we consider the trace operator from spaces $\mathfrak{B}_{p, q}^{s}\left(\mathbb{R}^{n}\right)$ into Lebesgue spaces $L_{p}(\Gamma)$, where $\Gamma$ is a $d$-set according to Definition 2.1. Furthermore, we obtain estimates for approximation numbers of the embeddings between function spaces of the above type.

We first introduce entropy numbers and collect some properties afterwards.

Let $X$ and $Y$ be quasi-Banach spaces and $T: X \rightarrow Y$ be a bounded linear operator. If, additionally, $T$ is continuous we write $T \in L(X, Y)$. Let

$$
U_{X}=\{x \in X:\|x \mid X\| \leq 1\}
$$


denote the unit ball in the quasi-Banach space $X$. An operator $T$ is called compact if for any given $\varepsilon>0$ we can cover the image of the unit ball $U_{X}$ with finitely many balls in $Y$ of radius $\varepsilon$.

Definition 3.1. Let $X, Y$ be quasi-Banach spaces and let $T \in L(X, Y)$. Then for all $k \in \mathbb{N}$, the $k$ th dyadic entropy number $e_{k}(T)$ of $T$ is defined by

$$
e_{k}(T)=\inf \left\{\varepsilon>0: T\left(U_{X}\right) \subset \bigcup_{j=1}^{2^{k-1}}\left(y_{j}+\varepsilon U_{Y}\right) \text { for some } y_{1}, \ldots, y_{2^{k-1}} \in Y\right\},
$$

where $U_{X}$ and $U_{Y}$ denote the unit balls in $X$ and $Y$, respectively.

These numbers have various elementary properties which are summarized in the following lemma.

Lemma 3.2. Let $X, Y$ and $Z$ be quasi-Banach spaces, let $S, T \in L(X, Y)$ and $R \in L(Y, Z)$.

(i) (Monotonicity) $\|T\| \geq e_{1}(T) \geq e_{2}(T) \geq \cdots \geq 0$. Moreover, $\|T\|=e_{1}(T)$, provided that $Y$ is a Banach space.

(ii) (Additivity) If $Y$ is a $p$-Banach space $(0<p \leq 1)$, then for all $j, k \in \mathbb{N}$

$$
e_{j+k-1}^{p}(S+T) \leq e_{j}^{p}(S)+e_{k}^{p}(T) .
$$

(iii) (Multiplicativity) For all $j, k \in \mathbb{N}$

$$
e_{j+k-1}(R T) \leq e_{j}(R) e_{k}(T) .
$$

(iv) (Compactness) $T$ is compact if, and only if,

$$
\lim _{k \rightarrow \infty} e_{k}(T)=0 .
$$

Remark 3.3. Entropy numbers in abstract and concrete quasi-Banach spaces have a long history which will not be repeated here. As for the general theory, including the use of entropy numbers in the spectral theory of compact operators, we refer to [3], [9] and [7]. Further information on the subject is also covered by the more recent books [4] and [1]. In particular, proofs of the above assertions may be found in $[4,1.3 .1]$.

Some problems about the entropy numbers of compact embeddings for function spaces can be transferred to corresponding questions in related sequence spaces. 
Let $d>0$ and $\left\{M_{j}\right\}_{j \in \mathbb{N}_{0}}$ be a sequence of natural numbers satisfying

$$
M_{j} \sim 2^{j d}, \quad j \in \mathbb{N}_{0} .
$$

At this stage, we want to study entropy numbers for the respective sequence spaces $b_{p, q}^{s, \varrho}\left(M_{j}\right)$, which are defined as the sequence spaces $b_{p, q}^{s, \varrho}$ in (1.8) with the sum over $m \in \mathbb{Z}^{n}$ replaced by a sum over $m=1, \ldots, M_{j}$. The assertions for compact embeddings between Besov spaces follow afterwards.

Proposition 3.4. Let $d>0,0<\sigma_{1}, \sigma_{2}<\infty$, and $0<q_{1}, q_{2} \leq \infty$. Furthermore, let $\varrho_{1}>\varrho_{2} \geq 0$,

$$
0<p_{1} \leq p_{2} \leq \infty \quad \text { and } \quad \delta=\sigma_{1}-\sigma_{2}-n\left(\frac{1}{p_{1}}-\frac{1}{p_{2}}\right)>0 .
$$

Then the identity map

$$
\text { id }: b_{p_{1}, q_{1}}^{\sigma_{1}, \varrho_{1}}\left(M_{j}\right) \rightarrow b_{p_{2}, q_{2}}^{\sigma_{2}, \varrho_{2}}\left(M_{j}\right)
$$

is compact, where $M_{j}$ is restricted by (3.1).

Proof. To prove our claim we use the decomposition

$$
\mathrm{id}=\sum_{j=0}^{\infty} \sum_{\beta \in \mathbb{N}_{0}^{n}} \mathrm{id}_{j \beta}
$$

where

$$
\operatorname{id}_{j \beta}(\lambda)=\left(0, \ldots, 0, \lambda_{j, 1}^{\beta}, \ldots, \lambda_{j, M_{j}}^{\beta}, 0, \ldots, 0\right)
$$

selects the $(j, \beta)$-th block, $j \in \mathbb{N}_{0}, \beta \in \mathbb{N}_{0}^{n}$ fixed. Taking $\lambda \in b_{p_{1}, q_{1}}^{\sigma_{1}, \varrho_{1}}\left(M_{j}\right)$, we see that

$$
\begin{aligned}
\left\|\operatorname{id}_{j \beta}(\lambda) \mid b_{p_{2}, q_{2}}^{\sigma_{2}, \varrho_{2}}\left(M_{j}\right)\right\| & =2^{\varrho_{2}|\beta|} 2^{j\left(\sigma_{2}-\frac{n}{p_{2}}\right)}\left(\sum_{m=1}^{M_{j}}\left|\lambda_{j, m}^{\beta}\right|^{p_{2}}\right)^{\frac{1}{p_{2}}} \\
& \leq 2^{\left(\varrho_{2}-\varrho_{1}\right)|\beta|} 2^{-j \delta} 2^{\varrho_{1}|\beta|} 2^{j\left(\sigma_{1}-\frac{n}{p_{1}}\right)}\left(\sum_{m=1}^{M_{j}}\left|\lambda_{j, m}^{\beta}\right|^{p_{1}}\right)^{\frac{1}{p_{1}}} \\
& \leq 2^{\left(\varrho_{2}-\varrho_{1}\right)|\beta|} 2^{-j \delta}\left\|\lambda \mid b_{p_{1}, q_{1}}^{\sigma_{1}, \varrho_{1}}\left(M_{j}\right)\right\|,
\end{aligned}
$$

where we have used $\ell_{p_{1}} \hookrightarrow \ell_{p_{2}}$ and (3.2) in the second step. 
Hence, for $\eta=\min \left(1, p_{2}, q_{2}\right)$, we calculate

$$
\begin{aligned}
\| \mathrm{id} & -\sum_{j=1}^{m_{1}} \sum_{|\beta| \leq m_{2}} \operatorname{id}_{j \beta} \mid b_{p_{2}, q_{2}}^{\sigma_{2}, \varrho_{2}}\left(M_{j}\right) \|^{\eta} \\
& \leq \sum_{j>m_{1}}^{\infty} \sum_{|\beta|>m_{2}}\left\|\operatorname{id}_{j \beta} \mid b_{p_{2}, q_{2}}^{\sigma_{2}, \varrho_{2}}\left(M_{j}\right)\right\|^{\eta} \\
& \leq \sum_{j>m_{1}}^{\infty} \sum_{|\beta|>m_{2}} 2^{\left(\varrho_{2}-\varrho_{1}\right)|\beta| \eta} 2^{-j \delta \eta} \longrightarrow 0 \quad \text { as } m_{1}, m_{2} \rightarrow \infty .
\end{aligned}
$$

Compactness now follows from the fact that id lies in the closure of continuous linear operators with finite rank, cf. [2, Section 4, Theorem 4.4 (c)].

The next theorem provides a sharp result for entropy numbers of the identity operator related to the sequence spaces $b_{p, q}^{s, \varrho}\left(M_{j}\right)$.

Theorem 3.5. Let $d>0,0<\sigma_{1}, \sigma_{2}<\infty$, and $0<q_{1}, q_{2} \leq \infty$. Furthermore, let $\varrho_{1}>\varrho_{2} \geq 0$,

$$
0<p_{1} \leq p_{2} \leq \infty \quad \text { and } \quad \delta=\sigma_{1}-\sigma_{2}-n\left(\frac{1}{p_{1}}-\frac{1}{p_{2}}\right)>0 .
$$

For the entropy numbers $e_{k}$ of the compact operator

$$
\text { id }: b_{p_{1}, q_{1}}^{\sigma_{1}, \varrho_{1}}\left(M_{j}\right) \rightarrow b_{p_{2}, q_{2}}^{\sigma_{2}, \varrho_{2}}\left(M_{j}\right)
$$

we have

$$
e_{k}(\mathrm{id}) \sim k^{-\frac{\delta}{d}+\frac{1}{p_{2}}-\frac{1}{p_{1}}}, \quad k \in \mathbb{N} .
$$

Remark 3.6. The proof of Theorem 3.5 follows from [15, Theorem 9.2]. Using the notation from this book we have

$$
b_{p_{i}, q_{i}}^{\sigma_{i}, \varrho_{i}}\left(M_{j}\right)=\ell_{\infty}\left[2^{\varrho_{i}} \ell_{q_{i}}\left(2^{j\left(\sigma_{i}-\frac{n}{p_{i}}\right)} \ell_{p_{i}}^{M_{j}}\right)\right], \quad i=1,2 .
$$

Recall the embedding assertions for Besov spaces $\mathscr{B}_{p, q}^{s}(\Gamma)$ defined via traces stated in Proposition 2.10. Since $d$-sets $\Gamma$ are compact, the related embeddings between diverse Besov spaces are also compact - at least in nonlimiting situations. This will follow from the next theorem when we study entropy numbers of corresponding embeddings.

We remark that the compactness of the embeddings between suitable spaces $\mathcal{B}_{p, q}^{s}(\Gamma)$ has nothing to do with the assumption that $\Gamma$ is a fractal with $|\Gamma|=0$ or a $d$-set with $d<n$. 
Theorem 3.7. Let $\Gamma$ be a $d$-set in $\mathbb{R}^{n}$ according to Definition 2.1 with $0<d<n$, $\mathcal{B}_{p, q}^{s}(\Gamma)$ be the spaces introduced in Definition 2.8, notationally complemented by $\mathcal{B}_{p, q}^{0}(\Gamma)=L_{p}(\Gamma)$, and $0<p, q \leq \infty$. Furthermore, let

$$
0 \leq s_{2}<s_{1}<\infty, \quad 0<p_{1}, p_{2} \leq \infty, \quad 0<q_{1}, q_{2} \leq \infty,
$$

and

$$
\delta_{+}=s_{1}-s_{2}-d\left(\frac{1}{p_{1}}-\frac{1}{p_{2}}\right)_{+}>0 .
$$

Then the embedding

$$
\text { id : } \mathscr{B}_{p_{1}, q_{1}}^{s_{1}}(\Gamma) \rightarrow \mathscr{B}_{p_{2}, q_{2}}^{s_{2}}(\Gamma)
$$

is compact and for the related entropy numbers we compute

$$
e_{k}(\mathrm{id}) \sim k^{-\frac{s_{1}-s_{2}}{d}}, \quad k \in \mathbb{N} .
$$

Proof. Step 1. Let $p_{2} \geq p_{1}$. With

$$
\sigma_{1}=s_{1}+\frac{n-d}{p_{1}}, \quad \sigma_{2}=s_{2}+\frac{n-d}{p_{2}}, \quad \text { and } \quad \delta=\delta_{+}
$$

we have

$$
\sigma_{1}-\frac{n}{p_{1}}=s_{1}-\frac{d}{p_{1}}=\delta+s_{2}-\frac{d}{p_{2}}=\delta+\sigma_{2}-\frac{n}{p_{2}} .
$$

Let $f \in \mathscr{B}_{p_{1}, q_{1}}^{S_{1}}(\Gamma)$, then by Definition 2.8 there is a (nonlinear) bounded extension operator $g=\operatorname{Ex} f$ such that

$$
\operatorname{Tr}_{\Gamma} g=f
$$

and

$$
\left\|g\left|\mathfrak{B}_{p_{1}, q_{1}}^{\sigma_{1}}\left(\mathbb{R}^{n}\right)\|\leq c\| f\right| \mathscr{B}_{p_{1}, q_{1}}^{s_{1}}(\Gamma)\right\| .
$$

We may assume that $g$ is zero outside a fixed neighborhood $\Lambda$ of $\Gamma$. Using the subatomic approach for $\mathfrak{B}_{p_{1}, q_{1}}^{\sigma_{1}}\left(\mathbb{R}^{n}\right)$, cf. Remark 1.4, we can find an optimal decomposition of $g$, i.e.,

$$
g(x)=\sum_{\beta \in \mathbb{N}_{0}^{n}} \sum_{j=0}^{\infty} \sum_{m \in \mathbb{Z}^{n}} \lambda_{j, m}^{\beta} k_{j, m}^{\beta}(x), \quad\left\|g\left|\mathfrak{B}_{p_{1}, q_{1}}^{\sigma_{1}}\left(\mathbb{R}^{n}\right)\|\sim\| \lambda\right| b_{p_{1}, q_{1}}^{\sigma_{1}, \varrho_{1}}\right\|
$$

with $\varrho_{1}>0$ large.

Let $M_{j}$ for fixed $j \in \mathbb{N}_{0}$ be the number of cubes $Q_{j, m}$ such that

$$
r Q_{j, m} \cap \Gamma \neq \emptyset .
$$


Since $\Gamma$ is compact it follows by Definition 2.1 that

$$
M_{j} \sim 2^{j d}, \quad j \in \mathbb{N}_{0} .
$$

This coincides with (3.1). We introduce the (nonlinear) operator $S$,

$$
S: \mathfrak{B}_{p_{1}, q_{1}}^{\sigma_{1}}\left(\mathbb{R}^{n}\right) \rightarrow b_{p_{1}, q_{1}}^{\sigma_{1}, \varrho_{1}}\left(M_{j}\right)
$$

by

$$
S g=\lambda, \quad \lambda=\left\{\lambda_{j, m}^{\beta}: \beta \in \mathbb{N}_{0}^{n}, j \in \mathbb{N}_{0}, m \in \mathbb{Z}^{n}, r Q_{j, m} \cap \Gamma \neq \emptyset\right\},
$$

where $g$ is given by (3.11). Recall that the expansion is not unique but this does not matter. It follows that $S$ is a bounded map since

$$
\|S\|=\sup _{g \neq 0} \frac{\left\|\lambda \mid b_{p_{1}, q_{1}}^{\sigma_{1}, \rho_{1}}\left(M_{j}\right)\right\|}{\left\|g \mid \mathfrak{B}_{p_{1}, q_{1}}^{\sigma_{1}}\left(\mathbb{R}^{n}\right)\right\|} \leq c .
$$

Next we construct the linear map $T$,

$$
T: b_{p_{2}, q_{2}}^{\sigma_{2}, \varrho_{2}}\left(M_{j}\right) \rightarrow \mathfrak{B}_{p_{2}, q_{2}}^{\sigma_{2}}\left(\mathbb{R}^{n}\right),
$$

given by

$$
T \lambda=\sum_{\beta \in \mathbb{N}_{0}^{n}} \sum_{j=0}^{\infty} \sum_{m=1}^{M_{j}} \lambda_{j, m}^{\beta} k_{j, m}^{\beta}(x) .
$$

It follows that $T$ is a linear (since the subatomic approach provides an expansion of functions via universal building blocks) and bounded map,

$$
\|T\|=\sup _{\lambda \neq 0} \frac{\left\|T \lambda \mid \mathfrak{B}_{p_{2}, q_{2}}^{\sigma_{2}}\left(\mathbb{R}^{n}\right)\right\|}{\left\|\lambda \mid b_{p_{2}, q_{2}}^{\sigma_{2}, q_{2}}\left(M_{j}\right)\right\|} \leq c .
$$

We complement the three bounded maps $\mathrm{Ex}, S, T$ by the identity operator

$$
\text { id }: b_{p_{1}, q_{1}}^{\sigma_{1}, \varrho_{1}}\left(M_{j}\right) \rightarrow b_{p_{2}, q_{2}}^{\sigma_{2}, \varrho_{2}}\left(M_{j}\right) \quad \text { with } \varrho_{1}>\varrho_{2},
$$

which is compact by Proposition 3.4 and the trace operator

$$
\operatorname{Tr}_{\Gamma}: \mathfrak{B}_{p_{2}, q_{2}}^{\sigma_{2}}\left(\mathbb{R}^{n}\right) \rightarrow \mathscr{B}_{p_{2}, q_{2}}^{s_{2}}(\Gamma),
$$

which is continuous by Definition 2.8. For $s_{2}=0$ we have $\sigma_{2}=\frac{n-d}{p_{2}}$ and may choose $q_{2}=\bar{p}_{2}$ according to (2.17). From the constructions it follows that

$$
\operatorname{id}\left(\mathcal{B}_{p_{1}, q_{1}}^{s_{1}}(\Gamma) \rightarrow \mathcal{B}_{p_{2}, q_{2}}^{s_{2}}(\Gamma)\right)=\operatorname{Tr}_{\Gamma} \circ T \circ \operatorname{id} \circ S \circ \operatorname{Ex} .
$$


The situation is illustrated in the following diagram:

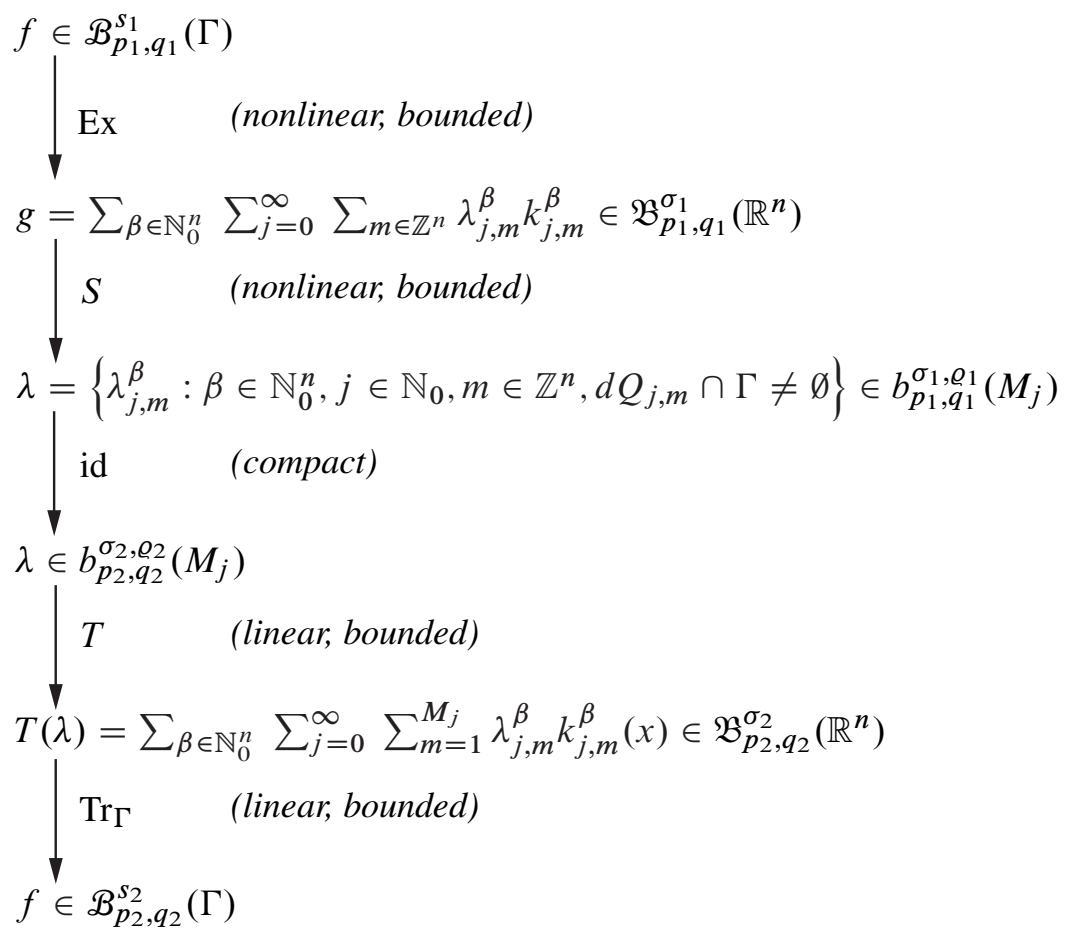

Hence, taking finally $\operatorname{Tr}_{\Gamma}$ we obtain $f$ by (3.10), where we started from. In particular, due to the fact that we have used the subatomic approach, the final outcome is independent of ambiguities in the nonlinear constructions Ex and $S$. The unit ball in $\mathscr{B}_{p_{1}, q_{1}}^{S_{1}}(\Gamma)$ is mapped by $S \circ$ Ex into a bounded set in

$$
b_{p_{1}, q_{1}}^{\sigma_{1}, \varrho_{1}}\left(M_{j}\right) .
$$

Since the identity operator id from (3.12) is compact, this bounded set is mapped into a pre-compact set in

$$
b_{p_{2}, q_{2}}^{\sigma_{2}, \varrho_{2}}\left(M_{j}\right),
$$

which can be covered by $2^{k}$ balls of radius $c e_{k}$ (id) with

$$
e_{k}(\mathrm{id}) \leq c k^{-\frac{\delta}{d}+\frac{1}{p_{2}}-\frac{1}{p_{1}}}, \quad k \in \mathbb{N} .
$$

This follows from Theorem 3.5, where we have used $p_{2} \geq p_{1}$. Applying the two linear and bounded maps $T$ and $\operatorname{Tr}_{\Gamma}$ afterwards does not change this covering assertion - using Lemma 3.2 (iii) and ignoring constants for the time being. Hence, 
we arrive at a covering of the unit ball in $\mathcal{B}_{p_{1}, q_{1}}^{s_{1}}(\Gamma)$ by $2^{k}$ balls of radius $c e_{k}(\mathrm{id})$ in $\mathcal{B}_{p_{2}, q_{2}}^{s_{2}}(\Gamma)$. Inserting

$$
\delta=s_{1}-s_{2}-d\left(\frac{1}{p_{1}}-\frac{1}{p_{2}}\right)
$$

in the exponent we finally obtain the desired estimate

$$
e_{k}(\mathrm{id}) \leq c k^{-\frac{s_{1}-s_{2}}{d}}, \quad k \in \mathbb{N} .
$$

Step 2. Let $p_{1}>p_{2}$. Since by Proposition 2.10,

$$
\mathcal{B}_{p_{1}, q_{2}}^{s_{2}}(\Gamma) \subset \mathcal{B}_{p_{2}, q_{2}}^{s_{2}}(\Gamma),
$$

we see that

$$
\mathcal{B}_{p_{1}, q_{1}}^{s_{1}}(\Gamma) \subset \mathcal{B}_{p_{1}, q_{2}}^{s_{2}}(\Gamma) \subset \mathcal{B}_{p_{2}, q_{2}}^{s_{2}}(\Gamma),
$$

and therefore (3.7) is a consequence of Step 1 applied to $p_{1}=p_{2}$. This completes the proof for the upper bound.

Step 3. The proof of the estimate from below,

$$
e_{k}\left(\mathrm{id}: \mathscr{B}_{p_{1}, q_{1}}^{s_{1}}(\Gamma) \rightarrow \mathcal{B}_{p_{2}, q_{2}}^{s_{2}}(\Gamma)\right) \geq c k^{-\frac{s_{1}-s_{2}}{d}}, \quad k \in \mathbb{N}
$$

is done in the same way as the corresponding estimate for the spaces $\mathbb{B}_{p, q}^{s}$, cf. [15, Theorem 20.6]. Proceeding indirectly, we assume that there is no such $c>0$ and we find a sequence $k_{j} \rightarrow \infty$ such that

$$
e_{k_{j}}\left(\mathrm{id}: \mathscr{B}_{p_{1}, q_{1}}^{s_{1}}(\Gamma) \rightarrow \mathcal{B}_{p_{2}, q_{2}}^{s_{2}}(\Gamma)\right) k_{j}^{\frac{s_{1}-s_{2}}{d}} \rightarrow 0 \quad \text { as } j \rightarrow \infty
$$

Using the estimate from above already established in Steps 1, 2 for $e_{k}$ (id) and the multiplication property for entropy numbers, cf. Lemma 3.2 (iii), we may assume that

$$
\mathcal{B}_{p_{2}, q_{2}}^{s_{2}}(\Gamma)=L_{p_{2}}(\Gamma),
$$

in particular, $s_{2}=0$ and $1<p_{1} \leq \infty$.

If $p_{2}<1$, by interpolation results of the $L_{p}$ spaces for any $f \in L_{p_{1}}(\Gamma)$ we have

$$
\left\|f\left|L_{p}(\Gamma)\|\leq\| f\right| L_{p_{1}}(\Gamma)\right\|^{1-\theta}\left\|f \mid L_{p_{2}}(\Gamma)\right\|^{\theta},
$$

where

$$
0<\theta<1 \text { and } \frac{1}{p}=\frac{1-\theta}{p_{1}}+\frac{\theta}{p_{2}} .
$$


Since entropy numbers behave well with respect to interpolation, in this context we refer to [4, Section 1.3.2], we obtain

$$
\begin{aligned}
e_{2 k_{j}} & \left(\mathrm{id}: \mathscr{B}_{p_{1}, q_{1}}^{s_{1}}(\Gamma) \rightarrow L_{p}(\Gamma)\right) \\
& \leq c e_{k_{j}}\left(\mathrm{id}: \mathscr{B}_{p_{1}, q_{1}}^{s_{1}}(\Gamma) \rightarrow L_{p_{1}}(\Gamma)\right)^{1-\theta} e_{k_{j}}\left(\mathrm{id}: \mathscr{B}_{p_{1}, q_{1}}^{s_{1}}(\Gamma) \rightarrow L_{p_{2}}(\Gamma)\right)^{\theta} \\
& \leq c^{\prime}\left(k_{j}^{-\frac{s_{1}}{d}}\right)^{1-\theta} e_{k_{j}}\left(\mathrm{id}: \mathscr{B}_{p_{1}, q_{1}}^{s_{1}}(\Gamma) \rightarrow L_{p_{2}}(\Gamma)\right)^{\theta},
\end{aligned}
$$

using the estimate from above for $e_{k}$ (id) in the last line. From (3.14) it follows that

$$
e_{2 k_{j}}\left(\mathrm{id}: \mathscr{B}_{p_{1}, q_{1}}^{s_{1}}(\Gamma) \rightarrow L_{p}(\Gamma)\right) k_{j}^{\frac{s_{1}}{d}} \rightarrow 0 \quad \text { as } j \rightarrow \infty .
$$

By (3.15) we may assume $p>1$. From these considerations it is clear that in order to prove the estimate from below it is sufficient to disprove the existence of a sequence $k_{j} \rightarrow \infty$ with

$$
e_{k_{j}}\left(\mathrm{id}: \mathscr{B}_{p_{1}, q_{1}}^{s_{1}}(\Gamma) \rightarrow L_{p_{2}}(\Gamma)\right) k_{j}^{\frac{s_{1}}{d}} \rightarrow 0,
$$

where $1<p_{1} \leq \infty$ and $1<p_{2} \leq \infty$.

Step 4. By Step 3 it remains to prove the following assertion: Let

$$
0<q \leq \infty, \quad 1<p_{1} \leq \infty, \quad 1<p_{2} \leq \infty, \quad s>d\left(\frac{1}{p_{1}}-\frac{1}{p_{2}}\right)_{+} .
$$

Then there is a constant $c>0$ such that

$$
e_{k}\left(\operatorname{id}: \mathscr{B}_{p_{1}, q}^{s}(\Gamma) \rightarrow L_{p_{2}}(\Gamma)\right) \geq c k^{-\frac{s}{d}}, \quad k \in \mathbb{N} .
$$

In this case the spaces considered coincide with the spaces in [16], cf. Remark 2.9 (iv). Therefore, the rest of the proof follows from [16, Theorem 20.6].

Remark 3.8. Assume that $0<q \leq \infty$ and

$$
s-d\left(\frac{1}{p_{1}}-\frac{1}{p_{2}}\right)_{+}>0 .
$$

Then (3.6) and (3.7) with $s_{2}=0$ can be rewritten in the form

$$
e_{k}\left(\operatorname{Tr}_{\Gamma}: \mathfrak{B}_{p_{1}, q}^{s+\frac{n-d}{p_{1}}}\left(\mathbb{R}^{n}\right) \rightarrow L_{p_{2}}(\Gamma)\right) \sim k^{-\frac{s}{d}}, \quad k \in \mathbb{N} .
$$

We give a different formulation of these results for entropy numbers in the following corollary. 
Corollary 3.9. Let $\Gamma$ be a $d$-set in $\mathbb{R}^{n}$ with $0<d<n$. Let $0<p_{1}, p_{2} \leq \infty$, $0<q \leq \infty$, and let

$$
s-\frac{n-d}{p_{1}}-d\left(\frac{1}{p_{1}}-\frac{1}{p_{2}}\right)_{+}>0 .
$$

The trace operator $\operatorname{Tr}_{\Gamma}$ of $\mathfrak{B}_{p_{1}, q}^{s}\left(\mathbb{R}^{n}\right)$ into $L_{p_{2}}(\Gamma)$ is compact and the related entropy numbers satisfy

$$
e_{k}\left(\operatorname{Tr}_{\Gamma}: \mathfrak{B}_{p_{1}, q}^{s}\left(\mathbb{R}^{n}\right) \rightarrow L_{p_{2}}(\Gamma)\right) \sim k^{-\frac{s}{d}+\frac{n-d}{d p_{1}}}, \quad k \in \mathbb{N} .
$$

There are previous results of this type for spaces $B_{p, q}^{s}\left(\mathbb{R}^{n}\right)$ and spaces with weights $B_{p, q}^{s}\left(\mathbb{R}^{n}, w(\cdot)\right)$ in [15, Section 20] and [10], respectively.

Finally, we give some estimates for approximation numbers.

Definition 3.10. Let $X, Y$ be quasi-Banach spaces, $T \in L(X, Y)$, and $k \in \mathbb{N}$. Then the $k$ th approximation number $a_{k}$ of $T$ is defined by

$$
a_{k}(T):=\inf \{\|T-L\|: L \in L(X, Y), \operatorname{rank} L<k\},
$$

where rank $L$ is the dimension of the range of $L$.

Our results are stated below.

Theorem 3.11. Let $\Gamma$ be a $d$-set according to Definition 2.1 with $0<d<n$. Furthermore, let $1<p<\infty, \frac{1}{p}+\frac{1}{p^{\prime}}=1$, and $\frac{n-d}{p}<s \leq \frac{n}{p}$. Then the trace operator

$$
\operatorname{Tr}_{\Gamma}: \mathfrak{B}_{p, p}^{s}\left(\mathbb{R}^{n}\right) \rightarrow L_{p}(\Gamma)
$$

is compact and for the related approximation numbers $a_{k}$ we have

$$
a_{k}\left(\operatorname{Tr}_{\Gamma}: \mathfrak{B}_{p, p}^{s}\left(\mathbb{R}^{n}\right) \rightarrow L_{p}(\Gamma)\right) \sim k^{-\frac{s}{d}+\frac{n-d}{d p}}, \quad k \in \mathbb{N} .
$$

Remark 3.12. Concerning the proof, the reader is referred to [17, Theorem 2, Remark 9]. Since $p>1$, the spaces considered in Theorem 3.11 coincide with the spaces under investigation in the mentioned paper.

Note that (3.18) coincides with (3.17) for $p_{1}=p_{2}=q=p$. One should expect a different behavior of $e_{k}\left(\operatorname{Tr}_{\Gamma}\right)$ and $a_{k}\left(\operatorname{Tr}_{\Gamma}\right)$ when $p_{1} \neq p_{2}$. At the moment this is outside our scope but gives a possibility for further investigation in the future. 


\section{Bibliography}

[1] B. Carl and I. Stephani, Entropy, Compactness and the Approximation of Operators, Cambridge Tracts in Mathematics 98, Cambridge University Press, Cambridge, 1990.

[2] J. B. Conway, A Course in Functional Analysis, 2nd edition, Graduate Texts in Mathematics 96, Springer-Verlag, New York, 1990.

[3] D. E. Edmunds and W. D. Evans, Spectral Theory and Differential Operators, Oxford Mathematical Monographs, Oxford Science Publications, The Clarendon Press, Oxford University Press, New York, 1987.

[4] D. E. Edmunds and H. Triebel, Function Spaces, Entropy Numbers, Differential Operators, Cambridge Tracts in Mathematics 120, Cambridge University Press, Cambridge, 1996.

[5] D. D. Haroske and C. Schneider, Besov spaces with positive smoothness on $\mathbb{R}^{n}$, embeddings and growth envelopes, J. Approx. Theory 161 (2009), no. 2, 723-747.

[6] L. I. Hedberg and Y. Netrusov, An axiomatic approach to function spaces, spectral synthesis, and Luzin approximation, Mem. Amer. Math. Soc. 188 (2007), no. 882.

[7] H. König, Eigenvalue Distribution of Compact Operators, Operator Theory: Advances and Applications 16, Birkhäuser Verlag, Basel, 1986.

[8] S. D. Moura, Function spaces of generalised smoothness, Dissertationes Math. (Rozprawy Mat.) 398 (2001).

[9] A. Pietsch, Eigenvalues and s-Numbers, Mathematik und ihre Anwendungen in Physik und Technik 43 [Mathematics and its Applications in Physics and Technology], Akademische Verlagsgesellschaft Geest \& Portig K.-G., Leipzig, 1987.

[10] I. Piotrowska, Entropy and approximation numbers of embeddings between weighted Besov spaces, in: Function spaces VIII, pp. 173-185, Banach Center Publ. 79, Polish Acad. Sci. Inst. Math., Warsaw, 2008.

[11] C. Schneider, On dilation operators in Besov spaces, Rev. Mat. Complut. 22 (2009), no. 1, 111-128.

[12] C. Schneider, Trace operators in Besov and Triebel-Lizorkin spaces, Z. Anal. Anwend. 29 (2010), no. 3, 275-302.

[13] C. Schneider, Traces in Besov and Triebel-Lizorkin spaces on domains, Math. Nachr. 284 (2011), no. 5-6, 572-586.

[14] H. Triebel, Theory of Function Spaces, Monographs in Mathematics 78, Birkhäuser Verlag, Basel, 1983.

[15] H. Triebel, Fractals and Spectra. Related to Fourier Analysis and Function Spaces, Monographs in Mathematics 91, Birkhäuser Verlag, Basel, 1997. 
[16] H. Triebel, The Structure of Functions, Monographs in Mathematics 97, Birkhäuser Verlag, Basel, 2001.

[17] H. Triebel, Approximation numbers in function spaces and the distribution of eigenvalues of some fractal elliptic operators, J. Approx. Theory 129 (2004), no. 1, 1-27.

[18] H. Triebel, Theory of Function Spaces, III, Monographs in Mathematics 100, Birkhäuser Verlag, Basel, 2006.

Received October 1, 2010.

\section{Author information}

Cornelia Schneider, Applied Mathematics III, University Erlangen-Nürnberg,

Haberstr. 2, 91058 Erlangen, Germany.

E-mail: schneider@am.uni-erlangen.de 